\title{
Competencias legales de los psicólogos clínicos
}

RESUMEN: Se analizan y estudian las competencias legales de los psicólogos clínicos en la red de servicios públicos de salud mental; derivadas de la aprobacion del Decreto de la Especialidad de Psicologia Clinica. El análisis se enmarca dentro de un modelo de atención comunitario apoyado en el trabajo del equipo interdisciplinario.

PALABRAS CLAVE: Psicología clínica, servicios de salud mental, equipo interdisciplinario

\section{Introducción.-}

El documento que presentamos a continuación es fruto del grupo de trabajo de la AEN que durante un año ha estado analizando y estudiando las competencias legales de los psicólogos clínicos en el marco de la AEN.

Nuestro objetivo ha partido del interés por intentar clarificar mediante la consulta de diversos documentos y legislaciones las competencias y funciones, que corresponden a los psicólogos clínicos como facultativos especialistas. Una vez oficializada la especialidad con la publicación del Decreto y la Orden Ministerial que lo operativiza, pensamos que es el momento de establecer lo más claramente sus competencias.

\begin{abstract}
The competences of clinical psychologists in the network of public mental health services are analyzed. These competences are derived from the Clinical Psychology Speciality Decree. The analysis is framed in a community attention model based on interdisciplinary team work.
\end{abstract}

KEY WORDS: Clinical Psychology, mental health services, interdisciplinary team work

Tales competencias perderían su sentido si no se contextualizan en referencia a un modelo determinado de atención. Este modelo parte de las normas generales de la Ley General de Sanidad de 1986 (1) y del Informe de la Comisión Ministerial para la Reforma Psiquiátrica de 1985 (2). En resumidas cuentas nos referimos al modelo sanitario que incorpora la atención de los trastornos en salud mental en el sistema sanitario general, a la territorialización de los servicios y a la descentralización de la atención del hospital psiquiátrico. El modelo de atención comunitaria que supone la comprensión integral del paciente, en sus aspectos biológicos y psicológicos dentro de un contexto 
social determinado. Tal modelo se apoya además, como elemento imprescindible, en el trabajo interdisciplinario hoy en boca de todo el mundo, pero que a nuestro juicio, con el influjo y la presión de la corriente ideológica actual, corre peligro de diluirse y perder su sentido. La Psicología Clínica ha contribuido en gran medida a su conceptualización, no solo aportando conceptos teóricos a la hora de entender el proceso salud-enfermedad desde una óptica diferente al modelo médico, sino además a través de la incorporación de los psicólogos al sistema sanitario, mediante la implementación de nuevas técnicas y abordajes clínicos para el tratamiento de los trastornos psíquicos.

Hoy en día asistimos bajo el título de "lo comunitario" a una perversión y distorsión del modelo. Cada vez con mayor frecuencia se impide a los psicólogos clínicos la realización de diagnósticos clínicos, indicaciones de ingresos hospitalarios, derivaciones, etc., con el argumento de que no están capacitados para diagnosticar el trastorno mental y con el pretexto de optimizar y clarificar las funciones de los equipos de salud mental. Tema que ya abordamos en un trabajo anterior con datos empíricos (3). La terminología es variada, optimización de funciones, jerarquización funcional, organización funcional según las responsabilidades clínicas, justamente en un momento en que con mayor claridad tales funciones están establecidas mediante la regulación de la Especialidad en Psicología Clínica. Ahora cuando la situación debería estar más clara se intentan difuminar tales competencias. El viejo “orden médico" vuelve de nuevo a filtrar bajo el aparente discurso de la profesionalización la jerarquía mas corporativista, la normativización más rígida, la exclusividad de funciones, la expulsión del campo clínico y técnico y en resumidas cuentas el pensamiento y el discurso único. Fuera de este "orden" está el caos. El discurso nos parece de sobra conocido. Frente a ello nos gustaría volver a plantear los desafíos de lo interdisciplinario, esto es, la articulación en pie de igualdad de distintos saberes y disciplinas, las distintas formas de entender un mismo hecho clínico y las dificultades de poderlo pensar en equipo.

Entendemos que en la atención a la salud mental las fronteras entre disciplinas nunca serán diáfanas, aunque la psicología clínica tiene su objeto y metodología propia, siempre habrá espacios comunes y precisamente el reto es poder compartirlos y diferenciar lo común y lo específico y enriquecernos con ello. Al tiempo sostenemos que el modelo no puede estar cerrado, tenemos la obligación de seguir pensando en su mejora, en los cambios necesarios para realizar una atención de calidad a las nuevas demandas que se nos plantean. No podemos tampoco dejar de lado los nuevos modelos de gestión que se están implantando, el modelo comunitario no se puede quedar en el pasado como algo obsoleto o romántico sin tomar en consideración las exigencias del sistema sanitario, de los gestores, la necesidad de medir la eficacia de los tratamientos, el coste de la atención, las nuevas tecnologías y las nuevas disciplinas.

Para hacer operativo, el presente trabajo lo hemos dividido en varios apartados que hemos considerado centrales 
en cuanto a funciones y competencias de los psicólogos clínicos, están establecidos en base a la documentación consultada.

\section{1.- Diagnósticos clínicos-evaluaciones}

En referencia al tema de si los psicólogos clínicos están o no capacitados para la realización de diagnósticos clínicos y evaluaciones, son muchos los documentos, tanto nacionales como internacionales, que confirman esta capacidad. En 1990, la Fiscalía General del Estado (4) solicitó un dictamen al Colegio Oficial de psicólogos sobre la capacitación de los psicólogos, para diagnosticar trastornos mentales de base no orgánica. El Colegio pidió a su vez informes a la AEN, a la Asociación Madrileña de Salud Mental y a los máximos responsables de los Servicios de S.M. de las Comunidades de Madrid, Navarra, Murcia, Andalucía y Asturias; así como a destacados catedráticos de la Universidad Española. Tanto el dictamen del Colegio como todos los informes solicitados se pronunciaron positivamente. Concretamente la AEN (4) manifestó que "los psicólogos clínicos están capacitados para hacer diagnóstico y tratamiento psicológico y además son profesionales imprescindibles en los Servicios de S.M., ya que su función es fundamental de cara a plantear tratamientos más integrales con los pacientes".

Asimismo la AEN sostenía, en su documento sobre las competencias de los psicólogos clínicos (5) (suscrito por AEPCP, ANPIR, el COP y la Sección de Psicología Clínica y de la Salud del COP ${ }^{1}$ ) que el hecho de que la labor asistencial esté a cargo de un equipo interdisciplinario, no supone que todo el mundo haga de todo, sino el abordaje desde diferentes disciplinas científicas del proceso saludenfermedad y del hecho psicopatológico específico que lleva al sujeto a la enfermedad. Tanto los psiquiatras como los psicólogos clínicos, poseen instrumentos teóricos y técnicos para diagnosticar, evaluar y tratar los trastornos mentales, algunos de ellos específicos y otros comunes.

Por su parte el Colegio Oficial de psicólogos, en su documento sobre perfiles profesionales del psicólogo (6), detalla en el apartado dedicado al perfil de la Psicología Clínica y de la Salud las funciones que actualmente desempeñan los psicólogos, entre las que figuran la evaluación, el diagnóstico y el tratamiento e intervención.

El mismo Insalud, en su documento sobre las normas de funcionamiento de las Unidades de S.M. (7), creadas a finales del 1985, especifica que estarán integradas por un equipo multidisciplinario y que las funciones asistenciales podrán ser efectuadas por cualquiera de los integrantes de la Unidad. Entre las funciones de los psicólogos figuran la atención especializada, psicoterapias, elaboración de informes psicológicos y psicodiag-nóstico.

Entre los documentos internacionales respecto a este tema nos encontramos, sólo por citar algunos, con el Dictionary of Occupational Tittles (8) y la Occupational Information Network (9) de Estados Unidos, la Clasificación Internacional de Ocupaciones de la OIT (10) y el informe de la Federación Europea de Asociaciones de Psicólogos (11). Todos estos documentos sostienen

\footnotetext{
${ }^{1}$ Asociación Española de Psicología Clínica y Psicopatología, Asociación Nacional de Psicólogos Internos Residentes, Colegio Oficial de Psicólogos, Sección de Psicología Clínica y de la Salud del Colegio Oficial de Psicólogos de Madrid.
} 
que el psicólogo clínico evalúa y diagnostica los trastornos mentales y emocionales de los individuos y aplica programas de tratamiento. Entre sus tareas se indican estudiar factores psicológicos en el diagnóstico, tratamiento y prevención de enfermedades mentales y trastornos emocionales o de la personalidad. Dada la naturaleza del trastorno mental, cualquiera de las dos profesiones, psicólogo clínico y psiquiatra, se encuentra suficientemente capacitado para el diagnóstico y el establecimiento de la estrategia terapéutica más conveniente en cada caso.

El Decreto de la Especialidad de Psicología Clínica (12) constituye al psicólogo clínico como Facultativo Especialista, capacitado para la realización de diagnósticos, evaluaciones y tratamientos de carácter psicológico. Es decir, con todas las competencias, funciones y obligaciones que corresponden a los facultativos especialistas. A raíz de su publicación en 1998, la Psicología Clínica ha sido reconocida por el Ministerio de Sanidad como especialidad sanitaria no médica, y el Ministerio de Educación la incluye en el organigrama de la Subdirección General de Especialidades en Ciencias de la Salud. El programa de la Especialidad aprobado por el Ministerio de Sanidad en 1996 (13), especifica entre sus objetivos generales capacitar a los psicólogos clínicos en formación, desde una perspectiva de atención integral, para el desempeño de las tareas de evaluación y diagnóstico, intervención y tratamientos psicológicos. Entre las habilidades que deben adquirir al finalizar la formación figura el diagnóstico y evaluación psicológica de los trastornos mentales, mediante técnicas de evaluación adecuadas (instrumentos y técnicas de entrevista).
La Subdirección General de Formación Sanitaria (14) precisa, además, la no exclusividad de la capacidad diagnóstica por parte de los psiquiatras al afirmar en referencia al Decreto de la Especialidad que "al incluir en el proyecto los términos de diagnóstico, evaluación y tratamiento, se hace calificándolos de carácter psicológico, sin que dichos términos pertenezcan en exclusiva a la profesión médica, ya que también es común su utilización en el ámbito de otras disciplinas. Asimismo, en la medida en que las actividades de diagnóstico y evaluación implican necesariamente la emisión de un juicio de valor, no cabe duda de que el psicólogo clínico, está capacitado para el enjuiciamiento (diagnóstico) de los trastornos psicológicos que se le presenten en el ejercicio de su profesión, con la finalidad de aplicar una posterior terapia psicológica (tratamiento), debiendo tener en cuenta que dicha capacidad de enjuiciamiento incluye también la posibilidad de derivar a un paciente al médico especialista en psiquiatría, cuando la patología atendida requiera la prescripción de fármacos".

Esta precisión queda finalmente ratificada por las sentencias del Tribunal Supremo $(15,16)$ a los recursos interpuestos por el Consejo General de Colegios Oficiales de Médicos y por las Sociedades de Psiquiatría Legal, de Psiquiatría y de Psiquiatría Biológica al Decreto de la Especialidad de Psicología Clínica, al desestimar los recursos. En ambas sentencias se sostiene la imposibilidad de otorgar con carácter exclusivo las competencias de diagnóstico y evaluación a psiquiatras ni a psicólogos; ya que cada especialidad tendrá que aplicar sus conocimientos en su ámbito científico propio. 
Por tanto nos parece que, el establecimiento del psicólogo clínico como facultativo especialista conlleva el reconocimiento de su capacidad de diagnóstico y evaluación, aclarando finalmente sus competencias legales y funciones clínicas (eso sí, no excluyentes). Como consecuencia, sería necesario que tales funciones quedaran claramente reflejadas en los diversos planes autonómicos de salud mental. En algunos de ellos, como en el de Valencia (17), se reservaba la valoración inicial de los pacientes exclusivamente al psiquiatra, hasta el momento en que los psicólogos tuvieran el reconocimiento de facultativos especialistas. Sin embargo en otras, como Navarra, ya habían reconocido al psicólogo como facultativo sanitario inmediatamente después de la publicación del real Decreto y en el 2000 como facultativo especialista, creando mediante decreto (18) el puesto de trabajo correspondiente, con las retribuciones establecidas por el Servicio Navarro de Salud para facultativos especialistas (19). Parece que la mayor o menor celeridad en el reconocimiento tiene que ver, sobre todo, con la voluntad política y con la asunción de un modelo asistencial apoyado en el trabajo interdisciplinario y en la corresponsabilidad clínica. Esperamos que en otros planes en los que todavía están por definir tales funciones, como es el caso del plan de salud mental de Madrid (20), se asuman los mismos principios.

\section{2.- Responsabilidad sobre los tratamientos}

En cuanto a la responsabilidad sobre los tratamientos queremos referirnos, en primer lugar, al tan discutido artículo 10 de la Ley General de Sanidad (1). Se refiere al derecho que tienen los sujetos a que se les asigne un médico que será su interlocutor principal con el equipo asistencial. Pero a continuación añade que, en caso de ausencia, otro facultativo del equipo asumirá tal responsabilidad. Y más adelante continúa diciendo que el paciente tendrá derecho a elegir el médico y los demás sanitarios titulados. La Carta de los Derechos del Paciente del Insalud (21) reproduce exactamente el mismo artículo. Es decir, el citado artículo asimila médico a facultativo, asimilación por otro lado que aparece contínuamente en casi todo el texto. Tendríamos que precisar que la Ley General de Sanidad es del año 1986, fecha en la que prácticamente los únicos facultativos que había en el Sistema Sanitario eran los médicos. Actualmente como todos sabemos hay muchos más, físicos, biólogos, radiofísicos hospitalarios y psicólogos clínicos. Este hecho está previsto en el propio Estatuto Jurídico del personal médico de la Seguridad Social (22) cuando se refiere a los profesionales que forman parte del personal que presta sus servicios, señalando que "quedan incorporados al Estatuto Jurídico del personal médico de la Seguridad Social todas las plazas correspondientes a las especialidades sanitarias legalmente reconocidas con independencia de la licenciatura universitaria requerida para la obtención del correspondiente título". Los Decretos posteriores $(23,24)$ sobre selección del personal estatutario recogen también el mismo articulado. Por tanto, extrapolar del citado texto que los médicos son los únicos responsables de los tratamientos parece un argumento sin demasiado fundamento. Supone ignorar la creación e incorporación de nuevas 
profesiones sanitarias no médicas y despreciar las aportaciones que han ido realizando en la atención a los ciudadanos. A pesar de todo ello queremos llamar la atención sobre las resistencias al cambio de la cultura sanitaria, ya que a pesar de la incorporación de nuevos profesionales especialistas, continúa equiparando facultativo a médico en textos legales de nueva creación, como la nueva Ley de la autonomía del paciente (25). Donde por un lado se reconoce la labor asistencial de los profesionales sanitarios y facultativos, al tiempo que se mantiene el viejo concepto médico de la responsabilidad sobre la salud del paciente.

Otros documentos y legislaciones posteriores han ido matizando la consideración del personal especializado, mediante el reconocimiento de sus competencias y funciones. Por ejemplo las sentencias del Tribunal Supremo, de 1990 (26), y del Tribunal Superior de Justicia del País Vasco, de 1989 y de 1990 (27), relativas a la exención del IVA correspondiente a los servicios prestados por los psicólogos en el ejercicio de su profesión. En ellas se precisa que frente a las profesiones sanitarias clásicas, como los médicos y farmacéuticos, existen otras que se han ido incorporando a este concepto, como ocurre con los biólogos, químicos y psicólogos. Considerando, de conformidad con la Ley General de Sanidad, que están integradas en el sistema sanitario general.

En el documento elaborado por la AEN y $\mathrm{AEPCP}^{2}$, sobre la inclusión de los psicólogos clínicos en Insalud (28), se reclama la ejecución de la disposición adicional segunda del Decreto de la Especialidad de Psicología Clínica, que establece la inclusión del personal estatutario que esté en posesión del título oficial de Psicólogo Especialista en Psicología Clínica y preste servicio en Instituciones Sanitarias de la Seguridad Social, en el Estatuto Jurídico de Personal Médico de la Seguridad Social. Siendo de esta forma reconocidos como facultativos especialistas, con sus correspondientes funciones, y sujetos a los derechos y obligaciones del citado estatuto. Como sabemos esta disposición no se ha incluido todavía, aunque sí se han ido incorporando al estatuto otras especialidades, como la de los radiofísicos hospitalarios, creada por Decreto en 1997. Las resistencias para asumir las modificaciones competenciales y legales de una nueva especialidad, como es la de Psicología Clínica, quedan patentes no sólo en este hecho, sino en las distintas interpretaciones que se pueden hacer respecto a la misma legislación. Por ejemplo, la que se desprende del informe de la Dirección de Salud Mental de Bizkaia (29), que ignora el Decreto de la Especialidad y la disposición a la que hacemos referencia. Aunque bien es cierto que reconoce que, un mero informe jurídico no puede dilucidar las diversas funciones y competencias entre psiquiatras y psicólogos y que se debería partir del "título habilitante". Cosa que como ya hemos señalado el mismo informe ignora.

Afortunadamente otras disposiciones sí toman en consideración la creación de la nueva especialidad. Por ejemplo el Decreto de la Comunidad autónoma de Galicia (30) relativo al personal sanitario, especifica que los psicólogos ejercerán su labor en el ámbito de atención especializada de salud,

\footnotetext{
${ }^{2}$ Asociación Española de Neuropsiquiatría, Asociación Española de Psicología Clínica y Psicopatología
} 
integrándose como facultativos superiores, siempre que ejerzan su trabajo dentro del ámbito de la salud pública y de la administración sanitaria. La Dirección de Ordenación Sanitaria del IASAM (31), en Andalucía, se pronuncia en el mismo sentido acogiéndose al Decreto de integración del AISN, que recoge por primera vez la figura del facultativo psicólogo. Afirmando incluso que el psicólogo debe incorporarse como facultativo especialista en los equipos del sistema de salud mental al lado del psiquiatra.

En esta misma línea la Federación Europea de Asociaciones de Psicólogos (11) señala que, dada la naturaleza del trastorno mental, tanto los psicólogos clínicos como los psiquiatras están suficientemente capacitados para establecer la estrategia terapéutica más conveniente en cada caso. Por lo tanto no tiene sentido establecer la preeminencia de uno sobre otro, sino que lo que se impone es la cooperación desde el reconocimiento de las distintas competencias. La Subdirección General de Formación Sanitaria del Ministerio de Sanidad (14) es muy clara en este sentido al señalar que no se puede admitir el planteamiento de configurar al psicólogo clínico como un profesional sin ningún ámbito de autonomía, cuyas actuaciones se harían depender, en todo caso, del previo diagnóstico y tratamiento médico, ya que ello implica desconocer la existencia, desde hace muchos años, de unos estudios universitarios de carácter superior centrados en la psicología.

El convenio sobre los derechos humanos del Consejo de Europa (32) aplica todo su articulado, sobre obligaciones profesionales y normas de conducta, referidas a todas las intervenciones practicadas con fines preventivos, diagnósticos, de tratamiento, rehabilitadores o de investigación, a médicos y profesionales de la salud en general, incluidos los psicólogos, cuya labor con los pacientes en unidades clínicas y de investigación puede tener profundos efectos. Queremos hacer hincapié en la importancia de este documento, que a diferencia de otras declaraciones internacionales, tiene carácter jurídico vinculante para los países que lo han suscrito. Entre ellos España donde ha entrado en vigor en enero de 2000.

No vamos a negar que, a pesar de que parece claramente fundamentada la capacidad para asumir la responsabilidad del tratamiento por parte del psicólogo clínico, determinadas líneas medicopsiquiátricas siguen y seguirán sustentando, contra toda evidencia, que la responsabilidad última de los tratamientos pertenece al psiquiatra. Así el borrador del perfil del psiquiatra (33) elaborado por la Asociación Holandesa de psiquiatría, plantea que "el psiquiatra es el responsable de la ejecución del conjunto del proceso de tratamiento del paciente psiquiátrico". Aunque reconoce la necesidad de cooperación con otras disciplinas para un diagnóstico y tratamiento eficaz. El documento parte de un método psiquiátrico ajustado, en todo momento, al modelo biomédico general, sin hacer ninguna distinción entre modelo médico y modelo clínico. Tema éste bastante cuestionado en la actualidad no solo por los psicólogos, sino por muchos de nuestros compañeros psiquiatras. El borrador fue contestado entre otras asociaciones por la 
INFORMES

AEN (34), que planteó que el liderazgo del equipo y la responsabilidad sobre el proceso terapéutico no tiene porqué ser una función exclusiva del psiquiatra, debiendo recaer en aquel profesional con mayor experiencia clínica, capacidad de liderazgo, etc. Diferenciando los requisitos de formación y la organización de las responsabilidades dentro de un equipo, de acuerdo a las ordenaciones sanitarias de cada país.

Las competencias y responsabilidades clínicas pueden consistir en responder del tratamiento y diagnóstico en su conjunto, en el caso de que el psiquiatra sea el coordinador del equipo, pero no pueden ser exclusivas, ya que otros especialistas, por ejemplo los psicólogos, precisamente por su propia disciplina, la Psicología Clínica, están capacitados para hacer lo mismo. Por lo tanto la responsabilidad clínica, dependiendo de cada situación, debe ser compartida por cada una de las disciplinas que intervengan en el abordaje de cada caso. Se debería enfocar la cuestión como una corresponsabilidad compartida dentro del equipo clínico.

\section{3.- Informes periciales judiciales, comisión} de tutela del menor, informes para otros especialistas e informes de IVE

En el tema de los informes nos encontramos con algunas situaciones ciertamente contradictorias. Según la Ley de Enjuiciamiento Civil (35), los psicólogos (al igual que otros profesionales con titulación oficial) pueden realizar informes periciales judiciales cuando la materia del juicio así lo precise. La Corte Suprema de EEUU, por ejemplo, reconoce desde 1959 a los psicólogos clínicos la capacidad legal de ejercer como expertos en la determinación de la enfermedad mental.

La Ley de Responsabilidad penal de los menores (36) establece que corresponde a los equipos técnicos adscritos a los juzgados y fiscalías de menores, en los que se integran los psicólogos forenses, la emisión de informes sobre la situación psicológica, educativa y familiar del menor, así como sobre su entorno social y en general sobre cualquier otra circunstancia relevante a los efectos de la adopción de alguna de las medidas cautelares previstas en la propia Ley. También podrán proponer una intervención socio-educativa o actividades de reparación o de conciliación con la víctima.

En relación a los informes requeridos por la Comisión de Tutela del Menor, el decreto sobre el procedimiento de constitución y ejercicio de la tutela y guarda del menor (37) señala que, en la instrucción del expediente podrán incorporarse al mismo cuantos informes de carácter social, sanitario, educativo y psicológico o cualquier otro que pueda aportar información útil para su resolución.

La realización de informes para otros especialistas, por parte de los psicólogos clínicos está incluida en muchos de los documentos que hemos mencionado con anterioridad: Las normas de funcionamiento de las Unidades de S.M. del Insalud (7), el informe de la Federación Europea de Asociaciones de Psicólogos (11), el convenio sobre los derechos humanos del Consejo de Europa (32), etc. Abundando en esta línea, la Subdirección de la Asesoría Jurídica del Servicio Andaluz de Salud (38)

\footnotetext{
${ }^{3}$ Interrupción Voluntaria del Embarazo
} 
INFORMES

dictamina que los psicólogos podrán elaborar y suscribir toda clase de informes, dictámenes o evaluaciones relacionados con su titulación y, en su caso, con su especialidad profesional. El informe emitido por el Colegio de Psicólogos de Andalucía, sobre las funciones del psicólogo clínico en el sistema sanitario (39), señala que, entre otras funciones, el psicólogo ha de realizar actividades de asesoramiento e interconsulta con otros profesionales. Finalmente en el Programa de la Especialidad de Psicología Clínica (13) figura, entre las habilidades que se deben adquirir en la formación, la realización de informes, certificados y peritajes psicológicos.

Cuestión diferente es la relativa a los informes de IVE. Como ya sabemos, en la emisión de los dictámenes exigidos para la práctica legal de la interrupción voluntaria del embarazo, en el tercer supuesto contemplado por la ley se exige como requisito que sea emitido por un médico de la especialidad, en este caso el psiquiatra. Pero a nuestro juicio merece la pena examinar la sentencia del recurso de casación del Tribunal Supremo de septiembre de 2001 (40) contra un delito de aborto a una psicóloga. Concretamente el voto particular de uno de los magistrados que reproducimos a continuación:. "El psicodiagnóstico es una de las competencias profesionales del psicólogo y comprende la valoración del comportamiento mediante la identificación de los procesos y sus alteraciones, discapacidades, disfunciones y trastornos. En el ámbito específico del trastorno mental, el psicólogo está plenamente capacitado para evaluar las alteraciones de índole psicológica y psico-social, así como en los de índole biológica, para cuya valoración se requiere la colaboración interdisciplinar del psicólogo". El magistrado menciona como parte de la prueba documental, "un amplio catálogo de resoluciones judiciales (sentencias, autos y providencias), en las que se pone de relieve la utilización de especialistas en Psicología, para dictaminar sobre aspectos de alteraciones de la personalidad y estado mental en los diversos órdenes jurisdiccionales. Afirmando que "toda esta documentación sirve para acreditar que la realidad social y profesional en la que se ven inmersos los psicólogos les puede llevar a considerar razonablemente que tienen capacidad técnica y preparación para diagnosticar los graves riesgos para la salud psíquica de la embarazada".

La línea argumental de este magistrado pone en primer plano las contradicciones legislativas que se vienen produciendo. Nos encontramos, por un lado, con el establecimiento de la Psicología Clínica como especialidad sanitaria y de los psicólogos clínicos como facultativos especialistas. Con el reconocimiento de su capacidad legal para realizar informes periciales judiciales, informes para la Comisión de Tutela del menor, etc., en base a los cuales se puede por ejemplo retirar o no la tutela de un menor, dictar medidas cautelares de orden penal, etc., pero, al tiempo, sin capacidad legal para realizar informes de IVE. Es claramente contradictorio el reconocimiento de dicha capacidad en unos órdenes y no en otros. Lo que, en resumidas cuentas, plantea la necesidad de revisar estos aspectos parciales de la legislación para adecuarlos a las funciones y competencias ya reconocidas en una amplia gama de resoluciones judiciales. 
4.- Indicaciones de ingresos y altas hospitalarias

Según la Ley de Enjuiciamiento Civil (35), el internamiento no voluntario por razones de trastorno psíquico requiere siempre de autorización judicial, salvo en los casos de urgencia, en cuyo caso es preceptivo dar cuenta cuanto antes al tribunal. Antes de conceder la autorización o ratificar el internamiento el tribunal deberá, entre otras medidas, oír el dictamen de un facultativo por él designado. En la misma resolución que acuerde el internamiento se expresará la obligación de los facultativos de informar periódicamente al tribunal sobre la necesidad de mantener la medida. En cuanto al alta, la Ley precisa que, cuando los facultativos que atiendan a la persona internada consideren que no es necesario mantener el internamiento, darán el alta al enfermo y lo comunicarán inmediatamente al tribunal competente (41). En relación con el internamiento de menores, tanto la Ley de Enjuiciamiento Civil (35) como la de Protección Jurídica del menor (42), especifican que requiere autorización judicial y se realizará siempre en un establecimiento de salud mental adecuado a su edad, previo informe de los servicios de asistencia al menor.

Por tanto, según la legislación española es el facultativo, entendemos que especialista en salud mental, es decir, psicólogo clínico y psiquiatra, el encargado de realizar dichos dictámenes e informes. A pesar de que a nuestro juicio la legislación es clara, se producen múltiples situaciones en la red de servicios de salud mental y en las unidades de ingreso hospitalarias, en donde se impide que los psicólogos clínicos realicen indicaciones de ingresos y altas y los informes pertinentes sin un fundamento claro.
El Draft White Paper Pshychiatry del Consejo de Ministros de Europa (43), que pretende establecer unas líneas comunes para todos los países europeos, esboza proposiciones contradictorias. Por ejemplo, para tomar una decisión sobre ingreso o tratamiento involuntario, plantea que el paciente debe ser examinado por un psiquiatra o médico con suficiente experiencia. Aunque más adelante señala que una autoridad independiente deberá ratificar o no la medida, variando en cada país europeo, pudiendo ser un juez, un médico autorizado, un asistente social o el gerente del hospital. Consideraciones que parecen sorprendentes, ya que en ningún momento se menciona al psicólogo clínico como capacitado para realizar tal examen, suponiendo que quizá pueda tener más experiencia en la materia un médico no especialista, al tiempo que se admite que pueda ser un asistente social quien ratifique la medida de internamiento. Similares consideraciones se plantean para el ingreso urgente. Por último en relación al establecimiento y control de los estándares de calidad, para la implementación de la legislación en salud mental, recomienda que los profesionales psiquiatras y no psiquiatras, así como abogados y usuarios se deben involucrar en el sistema para establecer y evaluar los niveles de calidad, para la implementación de la legislación en salud mental. Indicaciones que a tenor de lo que se sostiene con anterioridad nos parecen bastante contradictorias, a menos que obedezcan a otros objetivos ajenos a la calidad asistencial, a la salvaguarda de los derechos de los pacientes y a la asistencia clínica. 
Quisiéramos reseñar algunos de los comentarios y propuestas de la AEN a este documento (44): "Entre los aspectos más criticados, señalamos el que sólamente se haga referencia a intervenciones médicas y psiquiátricas, sin que se considere la intervención de los otros profesionales cualificados para efectuar evaluaciones e intervenciones terapéuticas, como son los psicólogos clínicos, que juegan un papel activo en la atención a estos pacientes $\mathrm{y}$ en el proceso de toma de decisiones, tales como el internamiento y el tratamiento involuntario. En cuanto a los procedimientos para tomar la decisión de ingreso y de tratamiento involuntarios consideramos adecuados los criterios de la legislación española”. Que se refieren a lo ya comentado anteriormente en referencia a la Ley de Enjuiciamiento Civil.

\section{5.- Bajas/altas laborales e incapacitación laboral}

La legislación sobre las bajas y altas laborales por incapacidad temporal, confirmación de las bajas, bajas definitivas por invalidez, etc $(45,46,47)$, establece, en primer lugar, que el control sanitario corresponde al sistema público. El parte médico de baja, expedido por el facultativo que lo realice, deberá consignar el diagnóstico, la descripción de la limitación en la capacidad funcional que motiva la situación de incapacidad temporal y la duración del probable proceso patológico. Incluyendo aquellos casos en los que haya que considerar las deficiencias de carácter físico o psíquico que anulen o modifiquen la capacidad, psíquica o sensorial, de quienes las padecen. Por lo tanto en el caso de un trastorno psíquico consideramos que, tanto el psicólogo clínico como el psiquiatra, están capacitados para realizar el informe clínico y la indicación correspondiente, ya que ambos tienen la consideración de facultativos especialistas. De esta forma y según señala la Ley General de la Seguridad Social (48), el Médico de Atencion Primaria o la Inspección Médica, en su caso, puede realizar la baja.

\section{6.- Incapacitación de enfermos con trastornos mentales \\ En este tema la Ley de} Enjuiciamiento Civil (35) señala, en cuanto a las pruebas y audiencias preceptivas en los procesos de incapacitación, que nunca se decidirá sobre la misma sin previo dictamen pericial médico, acordado por el tribunal. No especifica qué tipo de informe pericial médico se necesita. Aunque la misma Ley, en el articulado que se refiere a la prueba de peritos señala, como hemos reflejado anteriormente, que los psicólogos pueden ser llamados como peritos cuando la materia del juicio así lo precise. En este caso no sabemos si es una contradicción de la propia ley o bien una laguna que deja en la ambigüedad la delimitación de una competencia. Por lo que de nuevo se plantea la necesidad de adecuar la legislación al establecimiento de las vigentes competencias.

7.- Criterios para el desempeño de las jefaturas de servicios y coordinación de equipos

En el informe sobre el psicólogo clínico en el sistema sanitario, del Colegio de Psicólogos de Andalucía (39), se señalan entre sus funciones las de 
favorecer y participar en las actividades de coordinación, necesarias para el desarrollo de las actividades y programas del equipo de salud mental. En cuanto al establecimiento y reconocimiento de estas funciones tenemos legislaciones y planteamientos diferentes. En el decreto sobre organización y funcionamiento de los servicios de salud mental de Asturias (49) figura que en los centros de salud mental podrá existir un coordinador de centro, designado por el Consejero de Sanidad a propuesta del Director Regional para la Reforma de la Asistencia de S.M., entre los profesionales titulados superiores adscritos al centro. En este mismo sentido se pronuncia la resolución de la Consejera de Salud de la Comunidad de Madrid, que en las bases de la convocatoria para las plazas de Jefe de Salud Mental de Distrito (50) requiere la licenciatura en Medicina y especialidad en Psiquiatría o la licenciatura en Psicología y cuatro años de experiencia profesional. Sin embargo, en otras comunidades nos encontramos con un no reconocimiento de esta función. En la Orden por la que se crea la guía de unidades infanto-juvenil de atención a la salud mental de Castilla-La Mancha (51), la coordinación del equipo es una función propia del psiquiatra. Sin embargo tenemos que puntualizar que la orden es de 1985, fecha en la que solo los psiquiatras tenían categoría de facultativos especialistas. La Asociación CastellanoManchega de Neuropsiquiatría y Salud Mental en asamblea celebrada en mayo de 2002 (52), acordó que también los psicólogos clínicos pueden acceder a las jefaturas de dichas unidades, en su actual condición de facultativos especialistas. Por lo que se pedirá a la Conserjería de Sanidad la modificación oportuna.
Si en determinadas comunidades se reconoce la capacidad para ejercer esta función de coordinación y en otras no, sólo puede obedecer al sostenimiento de diferentes modelos de atención y no tanto a la calidad del sistema. Nos encontramos de nuevo con el modelo que pretende la resolución de la discriminación de funciones y responsabilidades mediante la jerarquización funcional, eufemismo utilizado para ejercer de forma unilateral la exclusividad de funciones de una disciplina.

En una línea parecida se pronuncia el ya citado Draft Psychiatrist's Profile (33) cuando sostiene que, "el psiquiatra dentro del contexto de la cooperación multidisciplinaria actúa como un "capitán" responsable del proceso de cuidados, la organización operacional se puede realizar si es necesario por otras disciplinas". Argumentando que el psiquiatra juega un rol central en la cooperación con otras disciplinas en el campo de la salud mental, porque es responsable del conjunto del tratamiento y de su implementación, lo cual permite el reconocimiento de la responsabilidad de otras disciplinas para ciertos aspectos del tratamiento, adjudicándole finalmente la responsabilidad de la eficacia de la cooperación entre disciplinas. Ante semejante nivel de responsabilidad, diríamos entre paréntesis que nos parece bastante omnipotente, no podemos por menos que sentirnos abrumados y lamentar que nuestros colegas tengan que sostener sobre sus hombros semejante tarea. La respuesta que la AEN (34) da sobre este tema nos parece suficientemente clara y ajustada a las competencias y funciones de psiquiatras y psicólogos clínicos: "Dentro 
de la formación básica del psiquiatra debe de haber habilidades de coordinación y liderazgo, pero esto no quiere decir que otros profesionales no puedan tener las mismas tareas y responsabilidades o dirigir un equipo. Este aspecto debería aparecer de una manera no excluyente con otros profesionales". En esta misma línea inciden otros documentos como el de perfiles profesionales del psicólogo del COP (6), que señala entre las funciones que desempeñan los psicólogos clínicos las de dirección, administración y gestión.

\section{Conclusiones}

No cabe duda que la Psicología Clínica como disciplina aporta un saber propio que contribuye a la salud mental de los individuos y el Estado capacita y certifica como profesionales idóneos para esta labor a los psicólogos clínicos.

De manera que los psicólogos clínicos tienen competencias y funciones claramente establecidas en la legislación y documentación consultada. Nos parece suficientemente clara la capacidad y competencia en el tema de los diagnósticos clínicos y evaluaciones, así como en el tema de la responsabilidad sobre los tratamientos. Otra cosa es que a pesar de todo ello se sigan produciendo dificultades que impiden a los psicólogos clínicos realizar tales funciones.

En cuanto a las competencias para realizar informes periciales judiciales, los solicitados por la Comisión de Tutela del Menor, los relativos a las bajas y altas y los realizados para otros especialistas, también parecen claramente establecidas. Únicamente en el caso de los informes de
IVE no es así. En este caso planteamos la necesidad de revisar y modificar la legislación vigente para adecuarla a las competencias de los psicólogos clínicos como facultativos especialistas que son.

Las competencias en cuanto a las indicaciones de ingresos y altas hospitalarias nos parecen también claramente especificadas en la legislación, como correspondientes a los facultativos especialistas.

Tema más complejo nos parece la cuestión relativa a la incapacitación de los pacientes con trastorno mental, en el que la ley sólo señala la necesidad del examen médico. Ya hemos señalado la ambigüedad de que no se especifique claramente este tema, a tenor del reconocimiento de la capacidad para emitir informes periciales judiciales por parte de los psicólogos. Nos parece que convendría adecuar también este aspecto para que sea consecuente a la oficialización de la Psicología Clínica.

En cuanto a la capacidad para coordinar equipos de salud mental, sólo recordar que en muchas Comunidades Autónomas está así reconocido y sería conveniente revisar por tanto las normativas en base a una coherencia legislativa.

Por último, los retos que se le plantean a la profesión del psicólogo clínico en el futuro inmediato pasan por exigir un exhaustivo cumplimiento del Real Decreto de la Especialidad y por el desarrollo de la Orden Ministerial que, sin duda, posibilitarán un claro establecimiento y consolidación del perfil del psicólogo clínico y la apertura de actuaciones en nuevos ámbitos de la salud, de la comunidad científica y de la formación clínica. 


\section{BIBLIOGRAFÍA}

(1) Ley 14/1986, General de Sanidad. BOE 29 de abril de 1986

(2) Ministerio de Sanidad y Consumo, Secretaría General Técnica, Servicio de publicaciones, Informe de la Comisión Ministerial para la reforma psiquiátrica, abril 1985.

(3) Ibáñez, M.; Alfaya, M.; Escudero, C.; Navarro, A.; Otero, C.; Pascual, P.y Suarez, V., "El reto del trabajo interdisciplinar: ¿hasta donde hemos llegado?", Revista de la AEN, 2001, julio/septiembre, 79. pp. 91-99

(4) Colegio Oficial de Psicólogos, Secretaría Estatal. Dossier sobre el dictamen emitido por el Colegio Oficial de Psicólogos, a petición de la Fiscalía General del Estado, sobre la capacitación y funciones de los psicólogos, septiembre 1990

(5) Asociación Española de Neuropsiquiatría, "Sobre las competencias de los psicólogos clínicos", Revista de la AEN, 1988, julio/septiembre, $\mathrm{n}^{\circ}$ 67, pp 175-176

(6) Colegio Oficial de psicólogos, Perfiles profesionales del psicólogo, Madrid, 1998

(7) Normas de funcionamiento de las Unidades de Salud Mental del Insalud, Papeles del Colegio, 1986, junio, vol. IV no 26, pp 22-25.

(8) Dictionary of Occupational Tittles. USA, 2001, ocupación 070.107-014

(9) Occupational Information Network, USA, 2001, ocupación 29-1066.00

(10) Organización Internacional del Trabajo, Clasificación Internacional de ocupaciones, Ginebra, 1991

(11) Federación Europea de Asociaciones Profesionales de Psicólogos (EFPPA). Informe a petición de la sala 3 del Tribunal Supremo sobre el campo profesional de la Psicología, Bruselas, 2001

(12) Real Decreto 2490/1998 de 20 de noviembre, por el que se crea y regula el título oficial de psicólogo especialista en Psicología Clínica. BOE de 2 de diciembre de 1998.

(13) Ministerio de Sanidad, Secretaría General Técnica, Servicio de Publicaciones, Programa de la Especialidad de Psicología Clínica. Guía de formación de Especialistas, 1996

(14) Subdirección General de Formación Sanitaria. Ministerio de Sanidad, Informe sobre el proyecto de Real Decreto de creación de la Especialidad de Psicología Clínica,1998

(15) Sentencia del Tribunal Supremo sobre el recurso contencioso-administrativo 49/1999, contra el Real Decreto de la Especialidad de Psicología Clínica. Octubre de 2002

(16) Sentencia del Tribunal Supremo sobre el recurso contencioso-administrativo 48/1999, contra el Real Decreto de la Especialidad de Psicología Clínica. Octubre de 2002

(17) Comunitat Valenciana, Plan director de salut mental i Assistencia Psiquiatrica, 2000.

(18) Decreto Foral de Navarra 625/1999, de 27 de diciembre, por el que se determinan y asignan las retribuciones a percibir por los psicólogos Especialistas en Psicología Clínica adscritos al Servicio Navarro de Salud. Boletín Oficial de Navarra ${ }^{\circ} 11$ de enero de 2000.

(19) Boletín Oficial de Navarra ${ }^{\circ} 157$, de diciembre de 1998. Anexo de estamentos y especialidades. Estamentos sanitarios

(20) Comunidad de Madrid, Consejería de Sanidad, Plan de Salud Mental, 2003-2008

(21) Carta de los Derechos del paciente del Insalud. Art. 10 de la ley General de Sanidad. BOE 29 de abril de 1986.

(22) Estatuto Jurídico del personal médico de la Seguridad Social, 1966. BOE de 23 de diciembre de 1966

(23) Decreto 118/1991, de 25 de enero, sobre selección de personal estatutario. BOE de 15 de febrero de 1991. 
INFORMES

(24) Decreto/Ley 1/1999, de 8 de enero, sobre selección de personal estatutario y provisión de plazas de Instituciones Sanitarias de la Seguridad Social. BOE de 9 de enero de 1999

(25) Ley 41/2002, básica reguladora de la autonomía del paciente y de derechos y obligaciones en materia de información y documentación clínica. BOE de 15 de noviembre de 2002.

(26) Sentencia del Tribunal Supremo sobre la exención del IVA que corresponde a los servicios prestados por los psicólogos en el ejercicio de su profesión. Diciembre 1990

(27) Sentencias del Tribunal Superior de Justicia del País Vasco sobre la exención del IVA que corresponde a los servicios prestados por los psicólogos en el ejercicio de su profesión. 1989 y 1990

(28) Asociación Española de Neuropsiquiatría y Asociación Española de Psicología Clínica y Psicopatología, "Para la adecuada inclusión de los psicólogos clínicos en el vigente estatuto jurídico del personal estatutario del Insalud", Revista de la AEN, 2000, octubre/diciembre, $n^{\circ}$ 76, pp 741-743

(29) Dirección de Salud Mental de Bizkaia, informe sobre cuestiones relacionadas con los psicólogos y médicos psiquiatras, 1994

(30) Decreto 303/1990 de 31 de Mayo, para la creación de la escala de personal sanitario al servicio de la Comunidad Autónoma de Galicia. Diario Oficial de Galicia de 8 de junio de 1990.

(31) Dirección de Ordenación Sanitaria del IASAM. Programa de formación postgraduada de psicólogos para la atención a la salud mental en Andalucía, 1990. Papeles del Colegio, 1990, febrero, no 43, pp 67-69.

(32) Consejo de Europa, Convenio relativo a los derechos humanos y la biomedicina. 1997. BOE de 20 de octubre de 1999.

(33) Dutch Psychiatric Association, Draft Psychiatrist's Profile, Agosto de 1996. Asociación Española de Neuropsiquiatría

(34) Asociación Española de Neuropsiquiatría, Comentarios y propuestas al perfil del psiquiatra. Acta de la Junta de Gobierno de la AEN, 30 y 31 de marzo de 2001

(35) Ley de Enjuiciamiento Civil de 2000. BOE de 8 de enero de 2000

(36) Ley de Responsabilidad Penal de los menores de 2000. BOE de 13 de enero de 2000.

(37) Decreto regulador del procedimiento de constitución y ejercicio de la tutela y guarda del menor. Consejera de integración Social de Madrid. BOE de 17 de mayo de 1992.

(38) Junta de Andalucía, Informe sobre competencias y funciones de los psicólogos en relación con la elaboración de informes y dictámenes profesionales, Noviembre de 2000

(39) Colegio de psicólogos de Andalucía, El psicólogo clínico en el sistema sanitario, 1989

(40) Segunda sentencia del recurso de casación 3068/1999 del Tribunal Supremo contra un delito de aborto a una psicóloga. Voto particular, de fecha 19 de septiembre de 2001

(41) Ministerio de Sanidad y Consumo y Consejo General del Poder Judicial, Actas de la jornada conjunta sobre Internamientos involuntarios. Intervenciones corporales y tratamientos sanitarios obligatorios.. Junio de 2000

(42) Ley de Protección Jurídica del Menor de 1996. BOE de 17 de enero de 1996

(43) Consejo de Ministros de Europa, Draft White paper on the protection of the human rights and dignity of people suffering from mental disorder, especially those placed as involuntary patients in a psychiatric establishment. Enero de 2000

(44) Asociación Española de Neuropsiquiatría, Comentarios y propuestas de la AEN al White paper. Acta de la Junta de Gobierno de la AEN, 6, 7 de octubre de 2000

(45) Ley 42/1994, de medidas fiscales y administrativas de orden social. BOE 31 de diciembre de 1994 
INFORMES

(46) Orden de 19 de junio de 1997, sobre incapacidad temporal. BOE 24 de junio de 1997

(47) Real Decreto 575/1997, de 18 de abril, por el que se modifican determinados aspectos de la gestión y control de la prestación económica de la Seguridad Social por incapacidad temporal. BOE de 24 de abril de 1997

(48) Ley 42/1994, General de la Seguridad Social. BOE de 30 de junio de 1994

(49) Decreto sobre organización y funcionamiento de los servicios de S.M. dependientes de la administración del principado de Asturias. B.O.P. 4 de julio de 1986

(50) Resolución de 2 de septiembre de 1994, para pruebas selectivas de puestos de Jefe de Salud Mental de Distrito. Consejera de Salud. BOCM de 20 de septiembre de 1994

(51) Orden de 15 de octubre de 1985, por la que se crea la guía de unidades infanto-juvenil de atención a la salud mental. Castilla-La Mancha. Diario Oficial de Castilla-la Mancha $n^{\circ} 42$ de 22 de octubre de 1985

(52) Acta de la asamblea extraordinaria de la A.C.M.N.S.M., de 15 de junio de 2002

* Coordinadora del grupo de trabajo. Psicóloga clínica. Coordinadora del Progama de S.M. de niños y adolescentes de Getafe

** Psicóloga clínica, Servicio de S.M. de Getafe

*** Psicólogo clínico, Sección Infancia y Familia del Servicio de Protección de Menores del Consell Insular de Mallorca

**** Psicóloga clínica, Servicio de S.M. de Navalcarnero

***** Psicóloga clínica, Jefe de Sección de Psicología del Servicio de S.M. del Hospital Provincial de Toledo

******* Psicóloga clínica. Equipo de S.M. de Soria

Fecha de recepción: 27-02-03 